\title{
Evaluation and
}

\section{management of a patient with possible cerebral venous thrombosis}

Fernando Barinagarrementeria, MD

Antonio Arauz, MD, PhD

\section{Summary}

Cerebral venous thrombosis (CVT) is a relatively rare form of stroke usually affecting young individuals. CVT is characterized by the diversity of its neurologic manifestations, which require a high level of clinical suspicion for diagnosis and prompt, appropriate treatment. Multiple circumstances have been associated with CVT, such as prior medical conditions, transient situations, certain medications, and some predisposing conditions. Headache, focal neurologic deficit, and seizures are the most frequent clinical manifestations at onset. MRI in combination with venography has become the imaging modality of choice, as this technique has a high sensitivity and specificity for establishing a diagnosis. CT venography is an alternative to MRI because this technique produces similar diagnostic results. Pharmacologic treatment of CVT with anticoagulants is widely accepted. Per 100 cases, the recurrence of $\mathrm{CVT}$ is $2.8 \%$ and the mortality of CVT is $10 \%$.
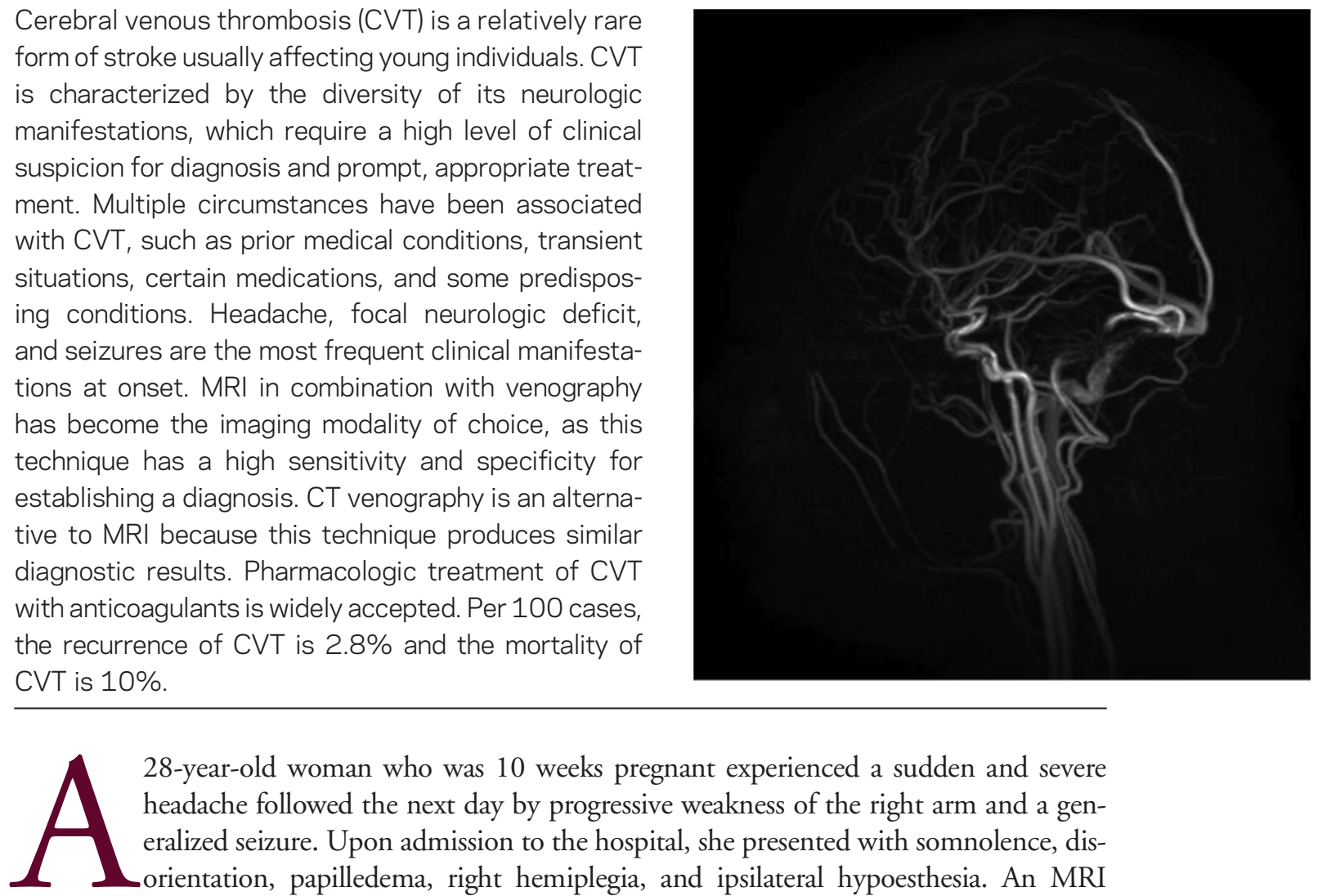

28-year-old woman who was 10 weeks pregnant experienced a sudden and severe headache followed the next day by progressive weakness of the right arm and a generalized seizure. Upon admission to the hospital, she presented with somnolence, disorientation, papilledema, right hemiplegia, and ipsilateral hypoesthesia. An MRI examination revealed a venous infarct in the left frontal area with hemorrhagic transformation and surrounding edema (figure 1). Magnetic resonance venography (MRV) demonstrated a thrombosis of the superior sagittal sinus (figure 2).

Division of Health Sciences (FB), Universidad del Valle de México, Quéretaro; and Stroke Clinic (AA), Instituto Nacional de Neurología y Neurocirugía Manuel Velasco Suárez, Colonia la Fama, México.

Funding information and disclosures are provided at the end of the article. Full disclosure form information provided by the authors is available with the full text of this article at Neurology.org/cp.

Correspondence to: antonio.arauz@prodigy.net.mx 
Figure 1 Venous infarct in the left frontal area with hemorrhagic transformation and surrounding edema

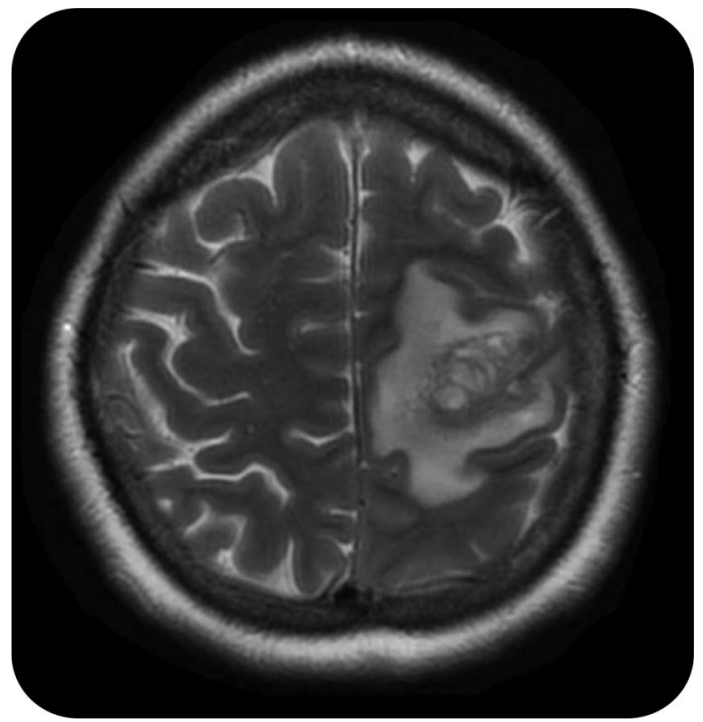

\section{When to suspect a diagnosis of CVT}

Cerebral venous thrombosis (CVT) is a relatively uncommon condition that accounts for approximately $1 \%$ of all strokes, and the majority of these cases occur in people younger than 50 years. ${ }^{1,2}$ In Mexico, in a prospective multihospital stroke registry, $3 \%$ of all cases were due to CVT. ${ }^{3}$

In young people, the main causes of CVT are related to pregnancy and puerperium and hereditary or acquired prothrombotic conditions, whereas cancer and prothrombotic conditions account for the majority of cases in older patients. ${ }^{1-4}$

The diagnosis of CVT is based on clinical suspicion and confirmation through imaging. Clinical findings are typically divided into 2 main categories depending on the mechanisms

Figure 2 Magnetic resonance venography demonstrating a thrombosis of the superior sagittal sinus

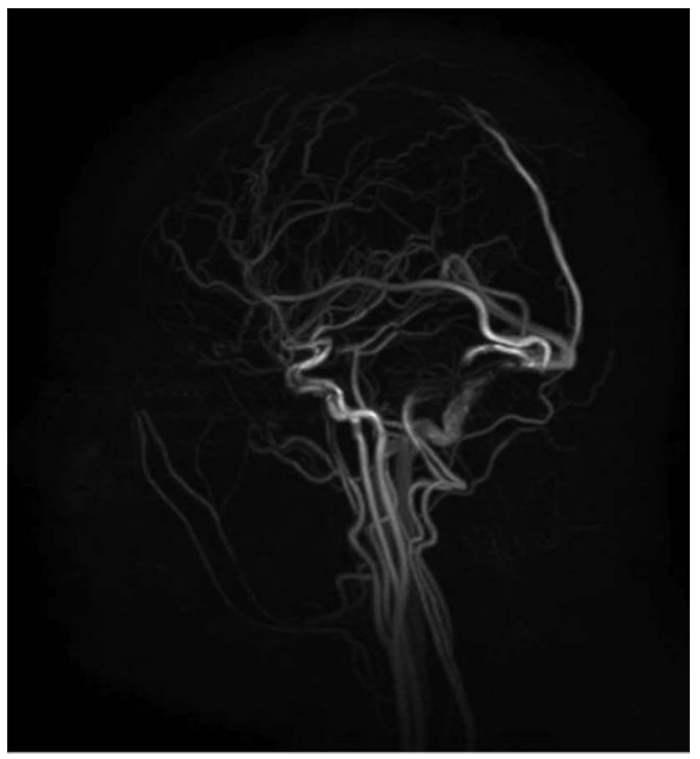




\section{The principal early signs of CVT on an MRI are the combination of an absence of a fluid void and altered signal intensity in the dural sinus.}

of neuronal dysfunction: they can be related to intracranial hypertension (ICH) secondary to impaired venous drainage or related to a focal brain injury from a venous infarct or hemorrhage. ${ }^{1,2,4-6}$ A diagnosis of CVT should be considered in cases such as the present one, during clinical conditions such as pregnancy or puerperium, and in patients using oral contraceptives (OC) or hormonal replacement therapy.

Headache is the most frequent symptom of CVT. In the largest cohort study (International Study on Cerebral Vein and Dural Sinus Thrombosis [ISCVT], which included 624 patients in 21 countries $^{3}$ ), headaches were reported by $90 \%$ of the patients. Headaches in CVT may appear suddenly, mirror a subarachnoid hemorrhage (SAH), or present as subacute. An isolated headache may be present in up to $25 \%$ of CVT cases, mainly in cases of lateral sinus thrombosis. ${ }^{5}$

Up to $40 \%$ of patients present with isolated ICH with clinical features such as headache and papilledema. ${ }^{5}$ Partial or generalized seizures occur in $40 \%$ of CVT cases. Another form of presentation, mainly in older people, is an encephalopathic form with mental disturbances and headaches. Patients with cerebral deep venous thrombosis usually display progressive neurologic deterioration. ${ }^{1,2,6}$ In our case, we did not find any prothrombotic conditions, and the patient had a typical clinical presentation with an acute headache, seizures, and papilledema.

\section{What are the main risk factors?}

There are multiple predisposing factors of CVT, including acquired risk factors and genetically based, inherited factors, such as thrombophilia. The most common risk factors include several prothrombotic conditions that account for between $21 \%$ and $34 \%$ of all cases. ${ }^{1,2,4-6}$ The causes and risk factors of CVT are detailed in table 1.

The use of OCs increases the risk for CVT, particularly in patients with prothrombotic conditions. ${ }^{1-4,6}$ However, the effects of hormones on coagulation variables appear to be modest, and some reports suggest that OC use could induce changes in both the procoagulant and anticoagulant pathways that may counterbalance each other. ${ }^{1,6,7}$ Pregnancy is a risk factor for stroke and is associated with significant changes in the hemostatic profile. Increases in the levels of coagulation factors occur due to increased protein synthesis driven by rising estrogen levels. Changes in the level of natural anticoagulants also occur; both total and free protein $S$ levels decrease progressively as gestation advances. ${ }^{7-9}$

The frequency of stroke subtypes during pregnancy and puerperium differs between countries. In Mexico, CVT was the most common subtype in a study of 240 patients experiencing stroke during pregnancy and puerperium. ${ }^{9}$ However, in Canada, arterial stroke occurred more commonly during pregnancy, and CVT was the most common stroke type during puerperium. ${ }^{8}$ In the United States, among 1,408,015 sampled deliveries from 1993 to 1994, there were 183 observed cases of peripartum stroke and 170 cases of peripartum CVT in 17 states according to data from the Healthcare Cost and Utilization Project. The factors that were significantly associated with an increased risk of both peripartum and postpartum CVT were cesarean delivery, hypertension, and infections other than pneumonia and influenza. ${ }^{10}$

In many obstetrics studies, CVT occurs more commonly during puerperium. Hypercoagulable states related to pregnancy persist after 4-6 weeks following labor. Anemia and dehydration are possible factors related to the predominance of these states. ${ }^{11}$

In older people with CVT, the presence of cancer should be considered. Thrombosis, a wellrecognized complication of cancer, may be the first manifestation of a malignancy. ${ }^{12}$ 
Table 1 Causes and factors associated with cerebral venous thrombosis (modified from Ehtisham and Stern ${ }^{16}$ )

Pregnancy and puerperium

Drugs

Oral contraceptives

Steroids

L-Asparaginasa

Hormone replacement

Malignant neoplasms

Visceral carcinomas, leukemia and lymphomas, myeloproliferative diseases

Systemic inflammatory diseases

Behçet disease

Wegener granulomatosis

Sarcoidosis

Arteritis of temporal artery

Infectious diseases

Bacterial: septicemia, endocarditis, typhoid, tuberculosis

Viral: measles, hepatitis, encephalitis, herpes, HIV, cytomegalovirus

Parasitic: malaria, trichinosis

Fungal: aspergillosis, cryptococcosis

Hematologic disorders

Polycythemia, paroxysmal hemoglobinuria, sickle-cell disease, posthemorrhagic anemia,

thrombocytopenia, increased lipoprotein (a)

Severe dehydration

Surgical procedures

Gastrointestinal diseases

Ulcerative colitis

Crohn disease

Liver cirrhosis

Heart disease

Congenital

Heart failure

Pacemaker

Others

Nephrotic syndrome

Exfoliative dermatitis

Homocystinuria

Local diseases

Extradural infections: mastoiditis, sinusitis, facial cellulitis, osteomyelitis, tonsillitis

Intradural/parenchymal: abscesses, empyema, meningitis

Tumors

Meningioma, metastasis, infiltration 


\section{Table 1 Continued}

Head injury

Jugular catheterization

Lumbar puncture

Others

Strokes and hemorrhages

Arteriovenous malformations

Arachnoid cyst

Internal jugular compression

Idiopathic

Coagulation disorders

Antithrombin III deficiency

$\mathrm{C}$ and $\mathrm{S}$ protein deficiency

Mutation of factor $V$ Leiden

Mutation of G20210 A prothrombin

Antiphospholipid antibodies

Lupus coagulant

Disseminated intravascular coagulation

Plasminogen deficiency

Cryofibrinogenemia

Hyperhomocysteinemia

Methylenetetrahydrofolate reductase mutation

Excess of factor VIII

Factor II mutation

Iron deficiency anemia

Folic acid, vitamin $\mathrm{B}_{6}$, and vitamin $\mathrm{B}_{12}$ deficiency

A hypercoagulable state occurs due to the ability of tumor cells to activate the coagulation system and secrete procoagulant/fibrinolytic substances and inflammatory cytokines. This state also occurs due to the physical interaction between tumor cells and vascular cells. ${ }^{12}$ The most common tumors related to thrombosis include mucin-secreting adenocarcinomas of the ovary, pancreas, and stomach; brain tumors; and hematologic malignancies. ${ }^{12}$

\section{What are the diagnostic studies?}

The first study that should be performed in a patient with suspected CVT is brain CT or MRI, which allow the physician to eliminate alternate diagnoses. CT and MRI also allow for the detection of parenchymal lesions, which could be caused by the thrombosis itself or by the rupture of dilated veins. ${ }^{13}$ Venous infarcts can undergo hemorrhagic transformation, and occasionally, even frank intracranial hemorrhages can be observed, including SAH. A hyperdense area of the cerebral venous sinus thrombi may be detected, but the CT will appear normal in up to $30 \%$ of cases. There are direct and indirect radiographic signs for the diagnosis of CVT (table 2). ${ }^{6,13}$ Direct signs are characterized by the presence of a thrombus in the affected vessel, whereas indirect signs are caused by brain parenchyma damage from ischemia as a consequence of venous flow obstruction. The most common direct sign is the dense triangle sign, which can be observed during the first 2 weeks of obstruction in up to 
Table 2 Direct and indirect signs of cerebral venous thrombosis on CT and MR

\begin{tabular}{|c|c|c|}
\hline & Indirect findings & Direct findings \\
\hline \multirow[t]{6}{*}{ Noncontrast } & $\begin{array}{l}\text { Nonhemorrhagic infarcts: can be cortical, subcortical } \\
\text { or deep; bilateral thalamic infarcts are highly } \\
\text { suggestive of deep vein thrombosis }\end{array}$ & Dense cord sign: represents a thrombosed cortical vein \\
\hline & Hemorrhagic venous infarcts (figure 1) & $\begin{array}{l}\text { Dense dural sinuses: represents an acute thrombosis of } \\
\text { the dural sinuses }\end{array}$ \\
\hline & Multifocal hemorrhages & $\begin{array}{l}\text { Dense jugular vein: represents an acute thrombosis of a } \\
\text { jugular vein }\end{array}$ \\
\hline & Subarachnoid hemorrhage & $\begin{array}{l}\text { Dense triangle or delta sign: observed as a dense } \\
\text { triangle (from a hyperdense thrombus); represents a } \\
\text { fresh thrombus of the superior sagittal sinus }\end{array}$ \\
\hline & Diffuse cerebral edema & \\
\hline & $\begin{array}{l}\text { Others: focal edema, mass effect, midline shift, and } \\
\text { hydrocephalus }\end{array}$ & \\
\hline Postcontrast & $\begin{array}{l}\text { Tentorial enhancement (most likely related to dural } \\
\text { venous collaterals), adjacent leptomeningeal } \\
\text { enhancement, and prominent cortical venous } \\
\text { enhancement due to venous congestion }\end{array}$ & Nonopacification of a filling defect in a dural sinus \\
\hline
\end{tabular}

$60 \%$ of the patients with an affected sagittal sinus. However, it is nonspecific, and falsepositives occur in patients with a raised hematocrit or with dehydration.

MRI combined with MRV is the most sensitive diagnostic tool for the detection of CVT. ${ }^{6,13}$ The signal intensity of a venous thrombus varies according to the time from the onset of thrombus formation. In the first week, a venous thrombus appears isointense on T1-weighted images and hypointense on T2-weighted images, whereas during the second week, a venous thrombus appears hyperdense on both T1- and T2-weighted images. The principal early signs of CVT on an MRI are the combination of an absence of a fluid void and altered signal intensity in the dural sinus. Currently, the most commonly used method for the diagnosis of CVT is MRV in conjunction with time-of-flight because it has excellent sensitivity to slow flow. ${ }^{13-16}$ CT venography (CTV) is another good option for the detection of CVT and is at least equivalent to MRV in the diagnosis of CVT. ${ }^{15}$

Digital subtraction angiography (DSA) is rarely used to diagnose CVT. However, DSA is useful in cortical vein thrombosis cases in which noninvasive imaging studies are inconclusive. ${ }^{16}$

To evaluate the recanalization of the occluded cortical veins or sinuses, a follow-up CTV or MRV at 3-6 months after diagnosis can be performed.

The presence of spontaneous CVT during pregnancy or puerperium makes the investigation of prothrombotic states necessary to determine the proper treatment and follow-up care, whereas elderly patients may require an evaluation for cancer.

\section{Therapeutic alternatives}

For treatment, the usual measures for handling ICH are recommended. Acetazolamide is a commonly used therapeutic alternative, ${ }^{16,17}$ although no controlled studies have demonstrated its usefulness. To prevent further seizures, early initiation of an antiepileptic is recommended in patients with a single seizure. ${ }^{16-18} \mathrm{~A}$ decompressive hemicraniectomy may be considered in patients with neurologic deterioration secondary to a severe mass effect or intracranial hemorrhage causing intractable ICH. ${ }^{16-18}$ The use of steroids to reduce vasogenic edema has not been shown to be beneficial and is associated with a worse prognosis in patients with 


\section{In cases of CVT associated with a transient}

risk factor, such as infection, trauma, or pregnancy, a treatment period of 3 months is sufficient.

parenchymal lesions. ${ }^{17,18}$ It is important to closely monitor the visual field and papilledema because prolonged pressure on the optic nerves can result in permanent blindness. Optic nerve fenestration is a treatment option that may halt progressive visual loss. ${ }^{17}$

Anticoagulants have become more widely used due to increasing evidence regarding their efficacy and safety. Heparin and oral anticoagulants (OA) are used to prevent thrombus growth, to facilitate recanalization, and to prevent a systemic embolism. Administration of anticoagulants is controversial because cerebral infarcts with hemorrhagic transformation or intracerebral hemorrhage are commonly present. ${ }^{14,17}$ Due to the rarity of CVT, clinical trials with anticoagulants are difficult to perform. ${ }^{14,17-19}$ To date, there are no precise instructions on which type of heparin to use. In the ISCVT study, ${ }^{2}$ unfractionated heparin (UFH) was used in approximately $75 \%$ of the cases. Some centers now prefer the use of low-molecular-weight heparin (LMWH) in therapeutic doses because it provides more steady anticoagulation and does not require dosage adjustment. For patients without contraindication, initial anticoagulation with adjusted dose UHF or LMWH followed by OA is a reasonable approach, regardless of the presence of an intracerebral hemorrhage. ${ }^{17-19}$ During pregnancy, as in our case, vitamin K antagonists are contraindicated. Guidelines recommend the continuation of LMWH throughout the pregnancy followed by continuation with OA for at least 6 weeks postpartum. ${ }^{14,17}$

In cases of CVT associated with a transient risk factor, such as infection, trauma, or pregnancy, a treatment period of 3 months is sufficient. In other conditions with a greater risk of recurrence, such as prothrombotic states, longer anticoagulation treatment is recommended. The current guidelines recommend treatment with OA for 3-6 months with a target international normalized ratio of 2-3 for patients with provoked CVT; for patients with unprovoked CVT, treatment with OA for 6-12 months is recommended. The role of new oral anticoagulants in CVT has not yet been defined; thus, their use is not recommended. ${ }^{14,17}$ However, despite aggressive anticoagulant therapy, $9 \%-13 \%$ of patients with CVT have a very poor outcome. ${ }^{17}$

\section{When is endovascular therapy indicated?}

Although it is clear that increasing venous outflow improves patient outcomes, it remains difficult to know when to choose this technique. The recent publication of a proposed treatment algorithm has provided some guidance where little evidence exists. ${ }^{20}$ This algorithm highlights the need to initially identify the underlying cause. It also indicates IV heparin or LMWH as a first-line treatment. Thrombolytic therapy should only be offered to patients who have not responded to anticoagulants or who have risk factors for a poor prognosis. Rheolytic thrombectomy, followed by thrombolysis to dissolve residual thrombi, seems to produce good patient outcomes. Despite this review, and because the current evidence with endovascular treatment is anecdotal and based on retrospective studies, it is not currently possible to extract conclusions about the benefit of this therapy. ${ }^{17,20}$

\section{DISCUSSION}

In our case, the patient was placed under treatment with lamotrigine and enoxaparin until the end of the pregnancy; following gestation, she recovered completely. After delivery, she continued with OA and anticonvulsive treatment. At the 1-year follow-up evaluation, an MRV showed complete recanalization of the sagittal sinus, and anticoagulation was discontinued. 


\section{REFERENCES}

1. Stam J. Thrombosis of the cerebral veins and sinuses. N Engl J Med 2005;352:1791-1798.

2. Ferro JM, Canhão P, Stam J, Bousser MG, Barinagarrementeria F; ISCVT Investigators. Prognosis of cerebral vein and dural sinus thrombosis: results of the International Study on Cerebral Vein and Dural Sinus Thrombosis (ISCVT). Stroke 2004;35:664-667.

3. Ruiz-Sandoval JL, Chiquete E, Bañuelos-Becerra LJ, et al. Cerebral venous thrombosis in a Mexican multicenter registry of acute cerebrovascular disease: the RENAMEVASC study. J Stroke Cerebrovasc Dis 2012;21:395-400.

4. Einhäupl KM, Villringer A, Haberl R. Clinical Spectrum of Sinus Venous Thrombosis. New York: Plenum; 1990.

5. Biousse V, Ameri A, Bousser MG. Isolated intracranial hypertension as the only sign of cerebral venous thrombosis. Neurology 1999;53:1537-1542.

6. Bousser MG. Cerebral venous thrombosis: diagnosis and management. J Neurol 2000;247:252-258.

7. Gosk-Bierska I, Wysokinski W, Brown RD Jr, et al. Cerebral venous sinus thrombosis: incidence of venous thrombosis recurrence and survival. Neurology 2006;67:814-819.

8. Jaigobin C, Silver FL. Stroke and pregnancy. Stroke 2000;31:2948-2951.

9. Cantú C, Arauz A, Aburto Y, Barinagarrementeria F, Ruiz S JL, Baizabal CJF. Cerebrovascular complications during pregnancy and postpartum: clinical and prognosis observations in 240 Hispanic women. Eur J Neurol 2011;18:819-825.

10. Lanska DJ, Kryscio RJ. Risk factors for peripartum and postpartum stroke and intracranial venous thrombosis. Stroke 2000;31:1274-1282.

11. Cantú C, Barinagarrementeria F. Cerebral venous thrombosis associated with pregnancy and puerperium: review of 67 cases. Stroke 1993;24:1880-1884.

12. Rogers LR. Cerebrovascular complications in patients with cancer. Semin Neurol 2004;24:453-460.

13. Wasay M, Azeemuddin M. Neuroimaging of cerebral venous thrombosis. J Neuroimaging 2005;15: $118-128$.

14. Liauw L, van Buchem MA, Spilt A, et al. EFNS guideline on the treatment of cerebral venous and sinus thrombosis in adult patients. Eur J Neurol 2010;10:1229-1235.

15. Khandelwal N, Agarwal A, Kochlar E, et al. Comparison of CT venography with MR venography in cerebral sinovenous thrombosis. AJR Am J Roentgenol 2006;187:1637-1643.

16. Ehtisham A, Stern BJ. Cerebral venous thrombosis: a review. Neurologist 2006;12:32-38.

17. Saposnik G, Barinagarrementeria F, Brown RD, et al. Diagnosis and management of cerebral venous thrombosis. Stroke 2011;42:1158-1192.

18. Coutinho JM, Stam J. How to treat cerebral venous sinus thrombosis. J Thromb Haemost 2010;8: 887-883.

19. Stam J, de Bruijn SF, de Veber G. Anticoagulation for cerebral sinus thrombosis. Cochrane Database Syst Rev 2002;CD002005.

20. Gala NB, Agarwal N, Barrese J, Gandhi CH, Prestigiacomo CJ. Current endovascular treatment options of dural venous sinus thrombosis: a review of the literature. J Neurointerv Surg 2013;5: $28-34$.

\section{STUDY FUNDING}

No targeted funding reported.

\section{DISCLOSURES}

F. Barinagarrementeria reports no disclosures. A. Arauz has received speaker honoraria from BoehringerIngelheim and Bristol Myers-Squibb. Full disclosure form information provided by the authors is available with the full text of this article at Neurology.org/cp.

\section{Related articles from other AAN physician and patient resources}

\section{Continuum ${ }^{\circledR}$ - ContinuumJournal.com}

Evaluation and Management of Cerebral Venous Thrombosis

April 2014;335-351.

Cerebrovascular Disorders Complicating Pregnancy

February 2014;80-99. 